\section{TARGETED THERAPIES}

\section{Apremilast reduces Behçet oral ulcers}

Treatment with apremilast $30 \mathrm{mg}$ twice daily for 12 weeks led to a greater decrease in the number of oral ulcers and in associated pain for patients with Behçet syndrome than treatment with placebo, according to the results of a new phase III study. More than half of the patients treated with apremilast (53\%) were free of oral ulcers at week 12 , compared with $22 \%$ in the placebo group, and the benefits of the treatment were apparent as early as week 1 .

Oral ulcers are an early and frequent manifestation of Behçet syndrome, and can have a substantial effect on a patient's quality of life. Although a number of agents are available to treat oral ulcers, including colchicine, topical or systemic glucocorticoids, TNF inhibitors or thalidomide, some patients fail to respond to these treatments. Apremilast, an orally available small-molecule phosphodiesterase 4 (PDE4) inhibitor that is already approved for the treatment of psoriasis and psoriatic arthritis, is a potential treatment for Behçet syndrome as it modulates several pro-inflammatory mediators that are upregulated in the disease (TNF, IL-2, IL-8, IL-17 and IFN $\gamma$ ).

The phase III results are consistent with those of a previous phase II trial of patients with Behçet syndrome from Turkey and the USA, but the current trial included a larger group of patients $(n=207)$ from 10 countries across 3 continents. Also notable is that patients in the phase III study had failed to respond to previous treatment with one or more nonbiologic agents.

Compared with the placebo group, patients in the apremilast group had a greater improvement in Behçet's Disease Quality of Life score, and in pain associated with oral ulcers as assessed on a $100-\mathrm{mm}$ visual analogue scale. However, adverse events, including diarrhoea, nausea and headache, were more frequent in the apremilast group than the placebo group.

As the trial had no active comparator, further studies are needed to determine how the efficacy of apremilast compares with that of other agents. Longer follow-up is also required to determine whether long-term administration of apremilast could be safe and effective.

Sarah Onuora

ORIGINAL ARTICLE Hatemi, G. et al. Trial of apremilast for oral ulcers in Behçet's syndrome. N. Engl.J. Med. 381, 1918-1928 (2019)

\title{
Short-term prednisolone improves hand OA
}

Inflammation is often present in the hand joints of patients with hand osteoarthritis (OA), particularly in those with erosive disease, and is linked with pain and disease progression. Targeting this inflammation with a short course of glucocorticoid therapy could offer pain relief, according to the results of the Hand Osteoarthritis Prednisolone Efficacy (HOPE) study.

The study specifically included patients with a flare of painful hand OA and signs of active synovial inflammation, in order to target those patients who would benefit most from the anti-inflammatory treatment. "More liberal patient inclusion criteria might have been a reason that previous trials (of other anti-inflammatory drugs) in hand OA produced negative results," suggests corresponding author Féline Kroon.

Compared with placebo, 6 weeks of treatment with oral prednisolone $10 \mathrm{mg}$ daily substantially reduced finger pain (as measured on a $100 \mathrm{~mm}$ visual analogue scale) and function (measured by AUSCAN function score and FIHOA score). Signs of inflammation, determined by MRI and ultrasonography, were also decreased. "The effects on pain and function we found in this trial exceeded those of all currently available treatment options," notes Kroon. Notably, symptoms returned to pre-treatment levels after the prednisolone was tapered over 2 weeks.

"The results of our study provide clinicians with a new short-term treatment option for patients with hand OA who report a flare-up of their disease," Kroon says. Whether continued treatment would have provided additional benefit or could eventually modify the disease course remains to be determined. In light of the potential complications of prolonged glucocorticoid therapy, further studies are needed to determine the optimal dosage and duration of treatment for inflammatory hand OA.

Sarah Onuora

ORIGINAL ARTICLE Kroon, F.P. B. et al. Results of a 6-week treatment with $10 \mathrm{mg}$ prednisolone in patients with hand osteoarthritis (HOPE): a double-blind, randomised, placebo-controlled trial. Lancet 394, 1993-2001 (2019)

\section{SPONDYLOARTHRITIS}

\section{New positive results for upadacitinib in AS}

The selective Janus kinase 1 (JAK1) inhibitor updacitinib, which was approved in 2019 for use in patients with rheumatoid arthritis (RA), is also showing promise as a treatment for ankylosing spondylitis (AS). In the SELECTAXIS 1 study, upadacitinib not only improved the signs and symptoms of AS, but also reduced inflammation in the spine and sacroiliac joints on MRI.

The study follows trials of upadacitinib in RA and also encouraging phase II trials of other JAK inhibitors in AS. SELECT-AXIS 1 enrolled adult patients with active AS who had not yet been treated with biologic DMARDs. A dose of $15 \mathrm{mg}$ updacitinib orally once daily was chosen for evaluation.

At week 14 of SELECT-AXIS 1, 52\% (48 of 93) of the patients treated with upadacitinib achieved an ASAS40 response (indicating $\geq 40 \%$ improvement from baseline according to Assessment of SpondyloArthritis International Society criteria), compared with $26 \%$ (24 of 94) of patients who were treated with placebo. Differences between the two groups in ASAS40 response were apparent as early as week 2 and sustained through 14 weeks.
As well as the primary endpoint of ASAS40 response, the study also met several secondary endpoints related to disease activity (including partial remission), the degree of functional limitation and MRI-detected axial inflammation. Updacitinib was well-tolerated, with a safety profile consistent with that seen in previous studies in RA. Rates of adverse events were similar in the treatment and placebo groups and no new safety concerns were apparent.

Long-term efficacy and safety data are expected to be collected in the ongoing extension period of SELECT-AXIS 1. Further studies are also needed to evaluate upadacitinib in patients with AS who have previously failed to respond to biologic DMARD therapy, as well as in the full spectrum of axial spondyloarthritis.

Sarah Onuora

ORIGINAL ARTICLE van der Heijde, D. et al. Efficacy and safety of upadacitinib in patients with active ankylosing spondylitis (SELECT-AXIS 1): a multicentre, randomised, double-blind, placebo-controlled, phase 2/3 trial. Lancet 394 2108-2117 (2019) 